\title{
Influence of radiating tube type on heat dissipation of assembled radiators
}

\author{
Ying Huang ${ }^{1 *}$, Lunjun Chen ${ }^{1}$, Meijun Li ${ }^{2}$, Bing Zhang ${ }^{2}$, Xiaoling Chen ${ }^{1}$, Linna Zhang ${ }^{1}$ \\ ${ }^{1}$ College of Mechanical Engineering, Guizhou University, Guiyang 550025, China \\ ${ }^{2}$ Yonghong Radiator Company, Guiyang 550009, China
}

Email:1954174359@qq.com

\begin{abstract}
The fabrication of assembled radiator features high production efficiency and causes no pollution to the environment thanks to the application of mechanical processing. Nevertheless, the application range of assembled radiator is greatly limited due to the low thermal efficiency. In the environment-friendly modern society, researchers are racking their brains to answer the difficult question of how to improve the thermal efficiency of assembled radiator to the level of brazed radiator. Against this backdrop, this paper compares the heat dissipation performance of flat tube type and wasp-waisted tube type automobile radiators through simulation and experiment, and discovers that wasp-waisted tube type assembled radiator has higher heat transfer capacity. It is proved in this research that the heat dissipation performance of assembled radiator can be improved by optimizing the shape of radiating tube, especially minimizing equivalent diameter and expanding the circulation area in the tube. Suffice it to say that this research provides valuable references for future study on the heat dissipation performance of assembled radiator.
\end{abstract}

Keywords: Assembled Radiator, Flat Tube Type, Heat Dissipation Performance, Wasp-waisted Tube Type.

\section{INTRODUCTION}

Radiating tube and radiating fin are two critically important radiator components in radiator. The structure of these components has an immediate impact on the radiator's assembly technology and heat transfer performance. From the structural perspective, there are mainly two strategies to improve the heat dissipation performance of assembled radiator. Strategy 1: improve the design of the radiating fin by optimizing the design of the louver to reduce the fin width, and seeking the optimal balance between thermal efficiency and resistance drop. Strategy 2: improve the radiating tube design. To this end, the equivalent diameter of the tube should be reduced to increase the heat transfer efficiency and circulation area in the tube, reduce resistance and enhance heat transfer. Besides, the material thickness should be minimized.

The design of improved radiating fin has become a focal point in the competition of automobile radiators. In terms of structural design, radiating fin is mainly divided into such three types as: flat fin, wavy fin and louvered fin. With an efficient heat transfer structure, the louvered fin represents the development trend of fin structure. The opening of the louver can effectively separate the air boundary layer and significantly improve the air side heat transfer efficiency. The louvered fin increases the heat dissipation performance by more than $20 \%$ from the level of flat fin. Among the various scholars probing into the louvered fin, V. P. Malapure et al. [1]carries out a numerical simulation of louvered fin type radiator and determines the form of louvered fin with the best heat transfer performance and the lowest flow resistance. S. H. Lee et al. [2] look for an efficient method to predict the heat transfer performance of a louver fin radiator in an automotive power system. R. Y. Wang et al. [3] examine the effects of the height and pitch of the louvered fin on the heat transfer coefficient and pressure drop. Q. W. Dong et al. [4] studied the influence of fin spacing, louver spacing and louver angle over the heat transfer and resistance of the flow channel. The numerous structural parameters add to the complexity of the research on the performance characteristics of louver. For example, parameters like louver spacing, louver length, fin spacing and fin thickness all have certain impacts on the heat transfer coefficient and pressure drop. Previous research has pointed out that: the heat transfer coefficient and pressure drop will fall simultaneously with the increase of louver spacing, fin spacing and fin height; by contrast, the two parameters will change in the opposite direction with the increase of the louver length.

Being a major determinant of radiator's heat transfer performance, the cross-section shape is a major entry point of the improvement of radiating pipe. In general, radiating pipe mainly takes the circular, elliptical and flat shapes. The circular and elliptical tubes are the dominant design in early products. By virtue of excellent manufacturability, cheap cost, small equipment investment and low entry threshold, the circular radiating tube has been extensively installed on lowemission cars. However, the circular tube has a poor heat transfer performance due to the small surface-area-to-volume 
ratio. The elliptical tube bears strong resemblance to the circular tube, except for two points. First, the heat dissipation performance is improved with a larger surface-area-tovolume ratio; second, the manufacturing technique is more complex despite the adoption of a similar tube arrangement. Through a numerical study of circular and oval tubes of approximately equal equivalent diameter and fin area, L. Zhang et al. [5] discover that the oval tube has a better heat dissipation effect due to the larger surface area.

The flat tube radiating pipe has been brought to the limelight for further improvement to the heat transfer efficiency of assembled radiator. In contrast to traditional circular tube, the flat tube has a larger heat transfer surface area, and better heat transfer efficiency. In addition, the flat tube requires much lighter weight and smaller size than the circular tube to transfer the same amount of heat. ES. Dasgupta et al. [6], D. Antonijevic[7], Y. Park et al. [8] and C. Cuevas et al. [9] explore the heat dissipation performance of flat tube louvered fin radiator. The results of their research echo those of numerous other scholars. X. Q. Ma et al. [10] conduct a comparative analysis on the heat dissipation performance of various forms of radiating tubes. Flat radiating tube is usually manufactured by pressing a circular tube with the same circumference (Figure 1). Studies have shown that, with the same Reynolds number Re, the flat tube outperforms the circular one in both total heat transfer coefficient and internal heat transfer coefficient.

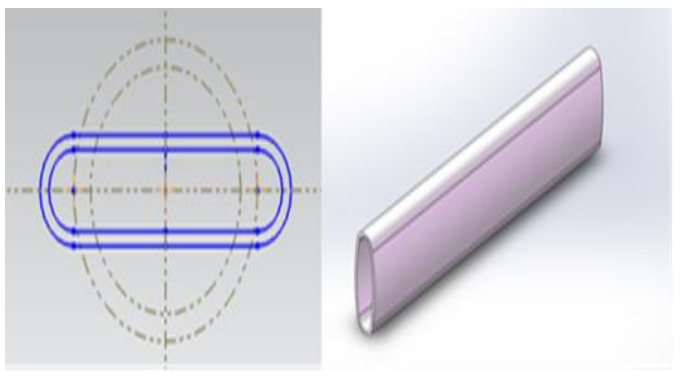

Figure 1. Sketch map of flat tube of radiator

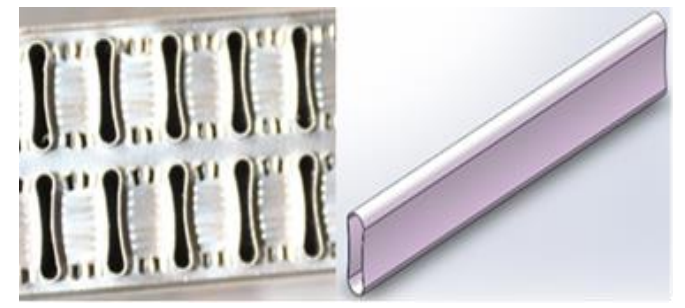

Figure2. Sketch map of waisted tube and fin of radiator

At present, flat radiating tube is applied to most assembled radiators. The tube enjoys a larger heat transfer surface area, and better heat transfer efficiency than the traditional circular tube. Moreover, it requires fewer weight and smaller size to transfer the same amount of heat than the circular tube. Unlike the traditional brazed radiator, assembled radiator causes no pollution or harm to the environment during fabrication. In the environment-friendly modern society, researchers are racking their brains to answer the difficult question of how to improve the thermal efficiency of assembled radiator to the level of brazed radiator. Based on the existing design of flat tube, this paper further optimizes the tube shape and develops a wasp-waisted type radiating tube, See Figure 2.The proposed tube symbolizes a brand new tactic in structural design: maximize the outer circumference and decrease the equivalent diameter without reducing the flow area.

\section{NUMERICAL SIMULATION ANALYSIS OF FLAT TUBE TYPE AND WASP-WAISTED TUBE TYPE ASSEMBLED RADIATORS}

The wide application of simulation software in radiator research has greatly facilitated scholars engaging in relevant fields. The heat dissipation of radiator goes as follows: as the high temperature liquid flows through the radiating tube, the heat is transferred to the surface of the fins through the heat conduction process between the tube wall and the fins; the cooling air passing through the flow channels between the louvered fins carries away the heat via the heat convection between the tube and the fin surface. This paper employs analysis software to simulate, analyze and compare the heat dissipation performance of flat tube type and wasp-waisted tube type assembled radiators under the same conditions. The two types of radiators share the same structure parameters, namely, base tube equivalent diameter, fin thickness, and fin surface area.

\subsection{Theoretical basis}

The radiator has a complex flow and heat transfer process. The fluid flow and heat transfer meet the laws of conservation of mass, energy, and momentum [11, 12, 13, 14].

Conservation of mass equation: The mass increase in fluid microelement per unit time is equal to the net mass which flows into the microelement in the same time interval.

$$
\frac{\partial \rho}{\partial t}+\frac{\partial(\rho \mathrm{u})}{\partial \mathrm{x}}+\frac{\partial(\rho \mathrm{v})}{\partial \mathrm{y}}+\frac{\partial(\rho \mathrm{w})}{\partial \mathrm{z}}=0
$$

Conservation of momentum equation: the change rate of the fluid in the microelement with respect to time equals the sum of various external forces acting on the microelement.

$$
\begin{aligned}
& \frac{\partial(\rho u)}{\partial t}+\operatorname{div}(p u u)=\operatorname{div}(\mu g r a d u)-\frac{\partial \rho}{\partial x}+S_{U} \\
& \frac{\partial(\rho v)}{\partial t}+\operatorname{div}(p v u)=\operatorname{div}(\mu g r a d v)-\frac{\partial \rho}{\partial x}+S_{V}
\end{aligned}
$$

$\frac{\partial(\rho w)}{\partial t}+\operatorname{div}(p w u)=\operatorname{div}\left(\mu_{\operatorname{grad}} w\right)-\frac{\partial \rho}{\partial x}+S_{W}$

Conservation of energy equation: The increase rate of the energy in the microelement is equal to the net heat flow into the microelement plus the work done by the body and surface forces on the microelement. The conservation of energy equation with temperature $\mathrm{T}$ as the variable is:

$\frac{\partial(\rho T)}{\partial t}+\operatorname{div}(p u T)=\operatorname{div}\left(\frac{k}{c_{p}} \operatorname{grad} T\right)-\frac{\partial \rho}{\partial x}+S_{T}$

Since a radiator involves a large number of heat transfer tubes and a complex fin structure, the computing power of existing computers is not enough to simulate the fluid flow in the radiator. Thus, the radiator model must be simplified to a 
certain extent.

\subsection{Simplification and establishment of geometric model}

The analysis model (Figure 3) is constructed with several equal-length radiating tubes consisting of 60 half-symmetrical radiating fins (fin spacing: $1 \mathrm{~mm}$ ). The air flows in the $\mathrm{x}$-axis direction. The inlet and outlet sections of the unit flow channel model are extended to ensure the uniform inlet flow rate. There is no reflux in the fluid at the outlet. Because the radiating fin is very thin and large in size, dividing the solid model of the radiator into grids would generate numerous grids and costs a lot of computing resources and time. To reduce computational load, the fin structure at the core is simplified to an area of porous medium during the simulation of airside heat transfer of the radiator $[15,16,17]$.

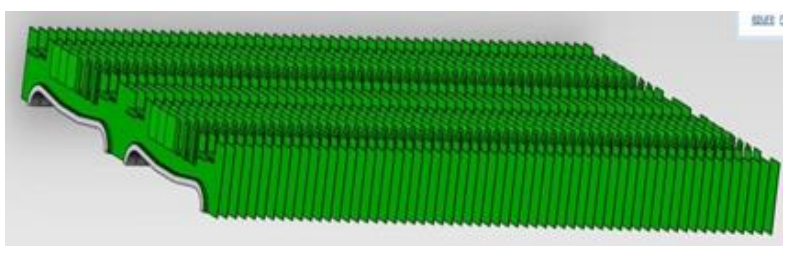

Figure 3. Simplified geometric model of wasp-waisted tube radiator

\subsection{Basic assumptions and boundary condition settings}

Water is used as the cooling liquid. Irrespective of the radiating fin structure, the radiating tube and radiating fins are modeled as an organic whole to eliminate thermal resistance. It is also assumed that the air is incompressible, the physical parameters of the air are constant, the air flow is a steady flow, the velocity direction of the air is vertical to the windward side, and the air is evenly distributed in the windward side. The impact of air gravity is ignored. The calculation area is extended in all directions than the actual area to ensure flow stability and the reliability of the physical model.

The boundary conditions are detailed as below:

1) The air inlet is set as the inlet boundary of velocity; the inlet air temperature: $293 \mathrm{~K}$.

2) The air outlet is set as the outlet boundary of pressure.

3) Inlet water temperature: $358 \mathrm{~K}$, water flow: $100 \mathrm{~L} / \mathrm{min}$.

4) Wind speed: $4 \mathrm{~m} / \mathrm{s}$.

5) The fluid is fully developed and evenly distributed in the flow channel.

6) The upper and lower surfaces are set as the periodic boundaries.

7) The symmetry plane is set as the symmetrical boundary.

8) The coupled heat transfer surface lies in the interface between the solid and the fluid regions.

\subsection{Results and analysis}

The simulated results of wasp-waisted tube type radiator and flat tube type radiator are explained with an example, in which the inlet air temperature is $293 \mathrm{~K}$, the wind speed is $4 \mathrm{~m} / \mathrm{s}$, the inlet water temperature is $358 \mathrm{~K}$, and the water flow is $100 \mathrm{~L} / \mathrm{min}$. The specific results are listed below. The outlet water temperature and water side pressure drop of the flat tube are $355.79 \mathrm{~K}$ and $2.399 \mathrm{~Pa}$, respectively (Figure 4 and Figure 5), while those of the wasp-waisted tube are $355.56 \mathrm{~K}$ and $2.589 \mathrm{~Pa}$, respectively (Figure 6 and Figure 7); the outlet wind temperature and wind side pressure drop the flat tube are $357.16 \mathrm{~K}$ and $359 \mathrm{~Pa}$, respectively (Figure 8 and Figure 9), while those of the wasp-waisted tube are $357.55 \mathrm{~K}$ and $399 \mathrm{~Pa}$, respectively (Figure 10 and Figure 11). The wasp-waisted tube has lower outlet water temperature and higher outlet wind temperature than the flat tube, indicating better heat transfer performance. It is mainly because the wasp-waisted shape disrupts the flow of air, causing obvious eddy currents in the near-wall region; the numerous eddy currents takes away more heat and enhances the local heat transfer in the region (Figure 12). However, the outlet pressure drops of the wasp-waisted tube type radiator are greater than those of flat tube type radiator at both the water side and wind side. The difference is explained as follows: since the outside wall of the flat tube is a plane surface, the air would flow at a uniform velocity; however, local turbulence would occur after the air flows around the radiating tube. On the contrary, the wasp-waisted tube has concave walls and longer outer circumference than the flat tube. The unique shape broadens the area of turbulence. In short, the air flow across the air channel of the wasp-waisted tube is much more chaotic than that of flat tube.

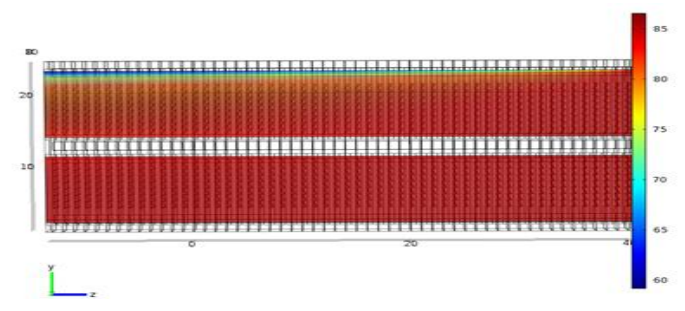

Figure 4. Water temperature distribution of flat tube

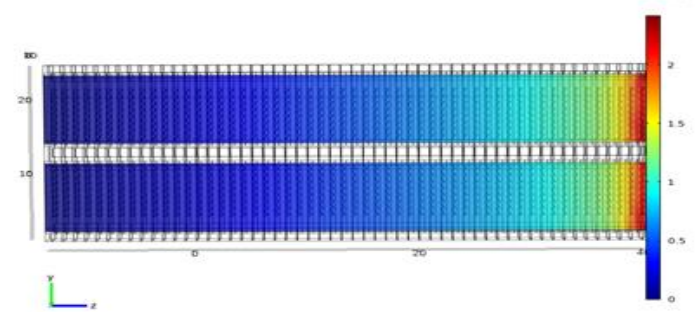

Figure 5. Water pressure distribution of flat tube

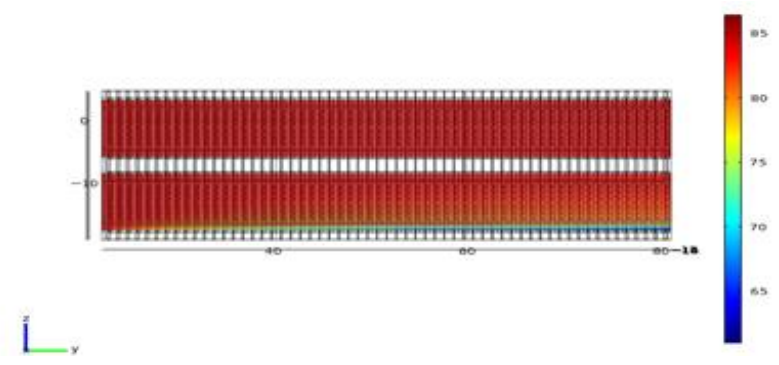

Figure 6. Water temperature distribution of wasp-waisted tube 


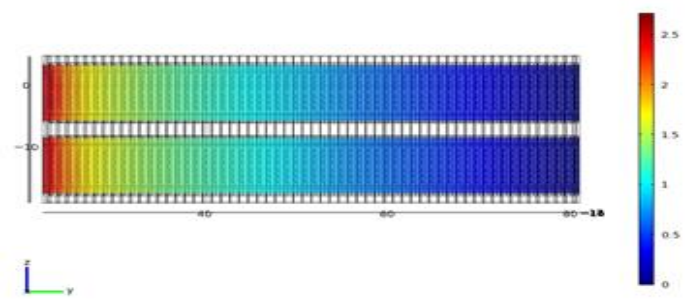

Figure 7. Water pressure distribution of wasp-waisted tube

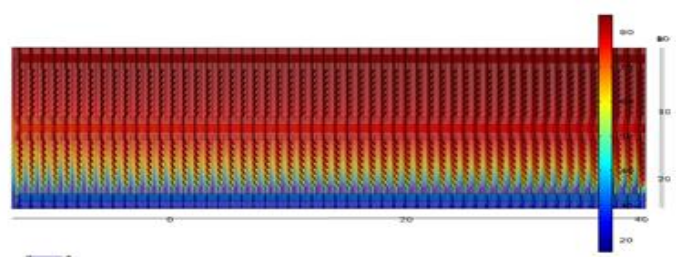

Figure 8. Temperature distribution of the fins on flat-tube

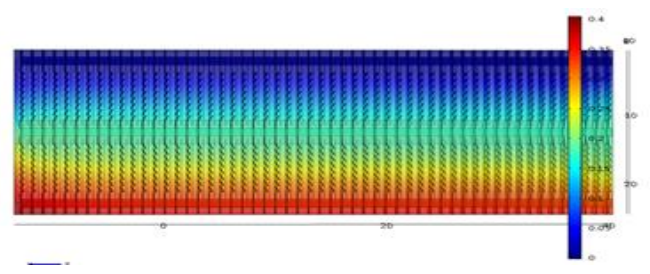

Figure 9. Air pressure distribution of the fins on flat-tube

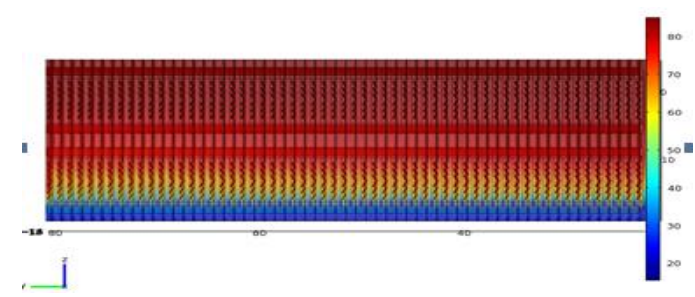

Figure 10. Temperature distribution of the fins on waspwaisted tube

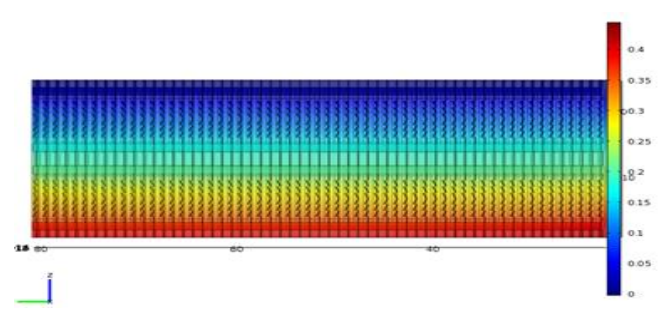

Figure 11. Air pressure distribution of the fins on waspwaisted tube

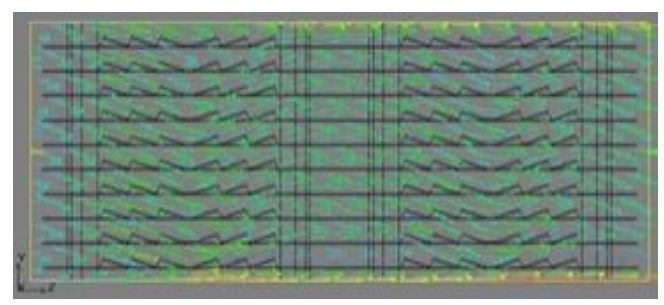

Figure 12. Cross section of air flow in wasp-waisted tube radiator

\subsection{Comprehensive performance evaluation}

The above analysis shows that the flat tube has good flow resistance with a low heat transfer coefficient and small pressure drop, while the wasp-waisted tube has good heat transfer performance with a high heat transfer coefficient and high pressure drop and flow resistance. It can be seen that the increase of heat transfer coefficient is bound to push up the pressure drop. This calls for a comprehensive evaluation standard for radiating tube performance. Hence, the comprehensive evaluation factor is introduced as the evaluation standard for radiating tube performance[18]. Considering the effects of heat transfer and pressure drop, the factor measures the energy transferred with the same amount of pump power. The greater the factor is, the better the overall performance. Since the wasp-waisted tube always boasts a higher comprehensive performance evaluation factor than the flat tube, it is proved that the wasp-waist structure can improve the comprehensive performance of radiating tube.

\subsection{Further optimization of wasp-waisted radiating tube}

Two models are constructed for wasp-waisted radiating tubes of different length-width ratios, aiming to reveal how different waist degrees affect the flow resistance and heat transfer characteristics of the radiating tube. The crosssections of the models are shown in the figure 13. Water is used as the cooling liquid, the walls of the radiating tube are regarded as the coupled heat transfer surface, and the air side is viewed as a porous medium. The heat transfer at the tube side is qualitatively analyzed without considering the specific structure of the fins. Through the simulation, it is found that the wasp-waisted tube increases with the waist degree but the growth rate gradually slows down.
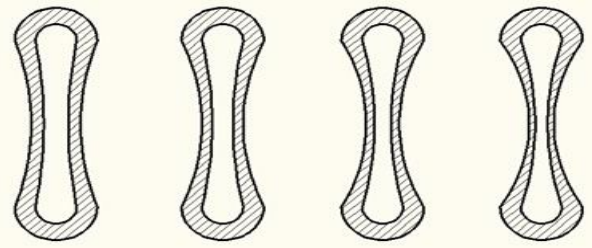

Figure 13. Sectional view of wasp-waisted tubes

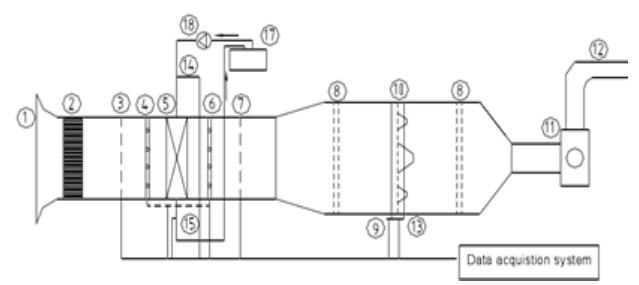

1. Air inlet; 2. Honeycomb straightener; 3.inlet temperature measuring station; 4. Pressure tap (inlet); 5. test unit; 6. pressure tap (outlet); 7. outlet temperature measuring station pressure tap (inlet); 8. setting means;

9. static pressure tap; 10 . multiple nozzle plate; 11 . variable exhaust fan system; 12. air outlet; 13 . difference pressure tap nozzle; 14 . inlet temperature tap water; 15 . outlet temperature tap water; 16 . data acquisition system; 17. hot water tank; 18. water pump.

Figure 13. Schematic diagram of the wind tunnel test apparatus 


\section{EXPERIMENTAL VERIFICATION AND ANALYSIS}

Wind tunnel test is an internationally recognized radiator test method. By adjusting the cooling air flow rate, water flow, etc., it can accurately measure radiator performance parameters, such as inlet/outlet temperature difference, inlet/outlet pressure drop and heat dissipating capacity. In this experiment, an artificial air flow channel is adopted to provide cooling air to the radiators. Major devices include air duct, circulating water circuit of the air duct, circulating water heating device, wind speed measuring instrument, temperature sensor, pressure sensor, mass flow meter and fans. The air duct creates the flow channel, and the fan provides power. Under the combined action of the air duct and the fan, the air flows through the radiator [19, 20]. A wind tunnel test is performed for each of the two types of radiators to compare their performance, and several charts are drawn to compare their heat dissipating capacity, wind resistance and water resistance. The test site is shown in Figure 13.

\section{TEST RESULTS AND ANALYSIS}

The experiment is conducted at room temperature. During the experiment, the ambient temperature is adjusted to $293 \mathrm{~K}$, and the inlet air temperature fluctuation is controlled within the range of $\pm 5^{\circ} \mathrm{C}$. There are no obstacles within the distance 3 times the air duct diameter from the inlet of the air duct. In total, five wind speed operating points are selected, respectively $2 \mathrm{~m} / \mathrm{s}, 4 \mathrm{~m} / \mathrm{s}, 6 \mathrm{~m} / \mathrm{s}, 8 \mathrm{~m} / \mathrm{s}$ and $10 \mathrm{~m} / \mathrm{s}$. Table 1 . shows that the two types of radiators have similar heat radiating capacity when the wind speed is at low levels; as the wind speed increases, the heat radiating capacity of waspwaisted tube climbs up because the tube generates more eddy currents than the flat tube. The trend is in good agreement with the results of numerical simulation.

Table 1. Heat dissipating capacity of radiator

\begin{tabular}{ccc}
\hline Wind speed(m/s) & wasp-waisted tube $(\mathrm{kw})$ & flat tube $(\mathrm{kw})$ \\
\hline 2 & 50.3 & 50.31 \\
4 & 60.87 & 60.63 \\
6 & 78.1 & 76.21 \\
8 & 91.71 & 88.65 \\
10 & 101.52 & 97.26 \\
\hline
\end{tabular}

Table 2. Wind resistance of of radiator

\begin{tabular}{ccc}
\hline Wind speed(m/s) & wasp-waisted tube(pa) & flat tube(pa) \\
\hline 2 & 93.86 & 101.99 \\
4 & 151.38 & 155.95 \\
6 & 291.68 & 307.57 \\
8 & 479.48 & 502.54 \\
10 & 686.77 & 733.99 \\
\hline
\end{tabular}

Table 3. Water resistance of radiator(kpa)

\begin{tabular}{ccc}
\hline Water flow rate( L/min) & wasp-waisted tube & flat tube \\
\hline 60 & 17.2 & 15.5 \\
87 & 39.8 & 37.6 \\
100 & 52.35 & 50 \\
\hline
\end{tabular}

As shown in Table 2., the wind resistance of both types of radiators increases with the wind speed. The wasp-waisted radiating tube has a lower wind resistance because the design of a larger fin area maximizes outer circumference and reduces the equivalent diameter. Furthermore, three water flow operating points are selected, respectively $60 \mathrm{~L} / \mathrm{min}$, $87 \mathrm{~L} / \mathrm{min}$, and $100 \mathrm{~L} / \mathrm{min}$, Table 3 . shows that the waspwaisted tube has larger water resistance than the flat tube. In light of the comprehensive performance evaluation factor, it is evaluated that the wasp-waisted tube type radiator is bless with better heat dissipation performance than that of the flat tube type radiator. The conclusion is consistent with the results of numerical simulation.

\section{CONCLUSION}

From the above simulation analysis and test results, it is concluded that the wasp-waisted tube type radiator has better comprehensive heat dissipation performance than the flat tube type radiator. The foremost reason lies in the size expansion of the radiating by the "wasp-waist" of the radiating tube. The waist structure induces eddy currents in the fluid, causes the separation of the critical layer of the tube wall, and thereby improves the heat transfer capacity of the whole product. The research opens up a new way to improve the heat dissipation performance of assembled radiator, i.e., to optimize the radiating tube by reducing the equivalent tube diameter and expanding the fin area, and to seek the optimal balance between thermal efficiency and resistance drop. For waspwaisted tube type assembled radiator with sufficient structural strength and pressure capacity, the heat dissipation performance increases with the waist degree in a certain range but the growth rate gradually slows down. It is necessary to conduct optimization analysis of various waist structures in the future so as to identify the waist degree for the optimal heat dissipation performance.

\section{ACKNOWLEDGMENT}

This study was supported by Project of Guizhou Province Science and Technology Program (20146004).

\section{REFERENCES}

[1] Malapure V.P., Mitra S.K., Bhattacharya A. (2007). Numerical investigation of fluid flow and heat transfer over louvered fins in compact heat exchanger, International Journal of Thermal Sciences, Vol. 46, pp. 199-211.

[2] Lee S.H., Hur N., Kang S. (2014). An efficient method to predict the heat transfer performance of a louver fin radiator in an automotive power system, J Mech Sci Technol, Vol. 28, No. 1, pp. 145-155. DOI: $\underline{10.1007 / \mathrm{s} 12206-013-0951-8}$

[3] Wang R.Y., Li J.X., Wu J.X. (2013). Optimization of louvered fin structure parameters of air side in radiators, Fluid Machinery, No. 6, pp. 74-78.

[4] Dong Q.W., Wang D., Liu M.S. (2012). Study on performance of automobile radiator with louvered fin, Machinery Design \& Manufacture, No. 1, pp. 115-117. Zhang L., Yang K., Liu W. (2009). Numerical simulation of flow and heat transfer in oval and 
circular finned tubes, Journal of Engineering Thermophysics, Vol. 30, No. 9, pp. 1571-1574.

[6] Dasgupta E.S., Askar S., Ismail M., Fartaj A., Quaiyum M.A. (2012). Air cooling by multiport slabs heat exchanger: an experimental approach, Exp Thermal Fluid Sci, Vol. 42, No. 10, pp. 46-54. DOI: 10.1016/j.expthermflusci.2012.04.009

[7] Antonijevic D. (2013). An engineering procedure for air side performance evaluation of flat tube heat exchangers with louvered fins, Heat Mass Transfer, Vol. 49, No. 1, pp. 117-127. DOI: 10.1007/s00231012-1073-Z

[8] Park Y., Jacobi A.M. (2009). Air-side heat transfer and friction correlations for flat-tube louver-fin heat exchangers, ASME J Heat Transf, Vol. 131, pp. 18011812.

[9] Cuevas C., Makaire D., Dardenne L., Ngendakumana P. (2011). Thermo-hydraulic characterization of a louv ered fin and flat tube heat exchanger, Exp Thermal Flu id Sci, Vol. 35, No. 1, pp. 154-1164. DOI: 10.1016/j.e xpthermflusci.2010.08.015

[10] Ma X.Q., Zhao J., Zheng M.Q., et al. (2015). Study on the effect of tube type on the heat transfer performance, Machinery Design \& Manufacture, No. 8, pp. 210-213.

[11] Huang Y., Chen L.J., Li M.J., Zhang B., Zhang L.N. (2016). Comparative study on the performance of flat tube type and wasp-waisted tube type radiators, International Journal of Heat and Technology, Vol. 34, No. 4, pp. 647-652. DOI: 10.18280/ijht.340414

[12] Tong Z.G., Wang Y.F., Chen D. (2014). Research on the structure optimization of automobile radiators, Energy Research and Information, Vol. 30, No. 2, pp. 108-112. DOI: $10.13259 /$ j.cnki.eri.2014.02.011

[13] Guo Q.J., Qi X.N., Wei Z., et al. (2016). 3D numerical simulation and analysis of refrigeration performance of the small diameter vortex tube, International Journal of Heat and Technology, Vol. 34, No. 3, pp. 513-520. DOI: $10.18280 /$ ijht.340324

[14] Pan X.F., Cao W.W., Liu Q.L., et al. (2014). Simulation research on car radiator fin, Journal of Shanghai University of Engineering Science, Vol. 28, No.3, pp. 193-197.

[15] Rafiee S.E., Sadeghiazad M.M. (2016). Heat and mass transfer between cold and hot vortex cores inside ranque-hilsch vortex tube-optimization of hot tube length, International Journal of Heat and Technology, Vol. 34, No. 1, pp. 31-38. DOI: 10.18280/ijht.340105
[16] Sayed A.S.A.E., Mesalhy O.M., Abdelatief M.A. (2015). Flow and heat transfer enhancement in tube heat exchangers, Heat Mass Transfer, Vol. 51, No. 1, pp. 1607-1630. DOI: $10.1007 / \mathrm{s} 00231-015-1669-1$

[17] Kim T.K., Kang H.C., Lee J.S. (2016). A porosity model for flow resistance calculation of heat exchanger with louvered fins, J Mech Sci Technol, Vol. 30, No. 4, pp. 1943-1948. DOI: 10.1007/s12206-016$\underline{0353-9}$

[18] Chang Y.J., Hsu K.C., Lin Y.T., et al. (2000). A generalized friction correlation for louver fin geometry, International Journal of Heat and Mass Transfer, Vol. 43, No. 12, pp. 2237-2243.

[19] Wu Y., Guo J.Z., Hu L., et al. (2016). Simulation and test research for the multi-structure of automotive louvered fins heat exchangers, Science Technology and Engineering, Vol. 16, No. 11, pp. 59-64.

[20] Tong Z.G., Wang Y.F., Chen D. (2014). Research on the structure optimization of automobile radiators, Energy Research and Information, Vol. 30, No. 2, pp. 108-112. DOI: 10.13259/j.cnki.eri.2014.02.011

\section{NOMENCLATURE}

t time

$\mathrm{U}$ the weight of the velocity vectors in the $\mathrm{x}$ direction

$\mathrm{V}$ the weight of the velocity vectors in the $y$ direction

$\mathrm{W}$ the weight of the velocity vectors in the $\mathrm{z}$ direction

$\mathrm{P} \quad$ the pressure on the fluid

Div the divergence of mathematical operator

Grad the gradient of mathematical operator

$\mathrm{Su}$ the generalized source terms of the conservation of momentum equation

Sv the generalized source terms of the conservation of momentum equation

Sw the generalized source terms of the conservation of momentum equation

$\mathrm{C}_{\mathrm{P}} \quad$ specific heat, $\mathrm{J} . \mathrm{kg}^{-1} \cdot \mathrm{K}^{-1}$

$\mathrm{T}$ temperature

$\mathrm{k}$ thermal conductivity, W. $\mathrm{m}^{-1} \cdot \mathrm{K}^{-1}$

$\mathrm{S}_{\mathrm{T}} \quad$ the internal heat source

\section{Greek symbols}

$\rho \quad$ Density, $\mathrm{kg} \cdot \mathrm{m}^{-3}$

$\mu \quad$ Dynamic viscosity, $\mathrm{kg} \cdot \mathrm{m}^{-1} \cdot \mathrm{S}^{-1}$ 